\title{
Serum PD-1 Levels Change with Immunotherapy Response but Do Not Predict Prognosis in Patients with Hepatocellular Carcinoma
}

\author{
Hye Won Lee $e^{1,2,3^{*}}$, Kyung Joo Cho ${ }^{1,3^{*}}$, Soon Young Shin ${ }^{1,3}$, Ha Yan Kim ${ }^{4}$, Eun Ju Lee ${ }^{4}$, Beom Kyung Kim ${ }^{1,2,3}$, \\ Seung Up Kim ${ }^{1,2,3}$, Jun Yong Park ${ }^{1,2,3}$, Do Young Kim ${ }^{1,2,3}$, Sang Hoon Ahn ${ }^{1,2,3}$, Kwang-Hyub Han ${ }^{1,2,3}$ \\ 'Department of Internal Medicine, Yonsei University College of Medicine, Seoul; ${ }^{2}$ Institute of Gastroenterology, Yonsei University College of \\ Medicine, Seoul; ${ }^{3}$ Yonsei Liver Center, Severance Hospital, Seoul; ${ }^{4}$ Biostatistics Collaboration Unit, Department of Biomedical Systems \\ Informatics, Yonsei University College of Medicine, Seoul, Korea
}

Received Feb. 1, 2019

Revised Feb. 26, 2019

Accepted Feb. 27, 2019
Background/Aims: Programmed death receptor 1 (PD-1) is a promising new target for treatment of patients with hepatocellular carcinoma (HCC). A high expression level of programmed death-ligand 1 (PD-L1) is a possible prognostic indicator for poor outcome in other malignancies. Here, we investigated the clinical significance of PD-1 and PD-L1 in patients with HCC.

Methods: We enrolled patients with HCC who underwent surgical resection at Severance Hospital between 2012 and 2017 and investigated the levels of PD-L1 in HCC tissues (tPD-L1) and PD-L1/PD-1 in serum (SPD-L1/SPD-1). We also aimed to determine whether expression levels correlated with clinical and histological features.

Results: A total of 72 patient samples were analyzed. The median SPD-L1 and SPD-1 levels were 25.72 and $341.44 \mathrm{pg} / \mathrm{mL}$, respectively. A positive correlation was detected between tPD-L1 and sPD-1 levels $\left(R^{2}=0.426, P<0.001\right)$. The median sPD-1 level increased linearly with $\mathrm{tPD}-\mathrm{L} 1$ score $(P=0.002)$. During the follow-up period, HCC recurred in eight $(11.1 \%)$ patients and liverrelated mortality occurred in eight (11.1\%) patients. Higher SPD-L1 levels $(\geq 19.18 \mathrm{pg} / \mathrm{mL})$ tended to be associated with liver-related mortality (hazard ratio $6.866 ; 95 \%$ confidence interval, 0.804-58.659, $P=0.078$ ). sPD-1 levels of patients treated with nivolumab as a second-line therapy changed serially, and $a>50 \%$ reduction in SPD-1 levels was observed immediately after nivolumab administration. However, SPD-1 level was not associated directly with prognosis in patients with advanced HCC.

Conclusions: The results demonstrated that PD-L1 and PD-1 levels changed according to the immunotherapy. However, no significant association with clinical outcome in patients with HCC was detected. (J Liver Cancer 2019;19:108-116)

Keywords: Programmed death-ligand 1; Programmed death receptor 1; Prognosis; Hepatocellular carcinoma

\section{Corresponding author : Kwang-Hyub Han}

Department of Internal Medicine, Yonsei University College of Medicine, 50-1 Yonsei-ro, Seodaemun-gu, Seoul 03722, Korea

Tel. +82-2-2228-1949, Fax. +82-2-312-7833

E-mail; gihankhys@yuhs.ac

https://orcid.org/0000-0003-3960-6539

\section{INTRODUCTION}

Programmed death receptor 1 (PD-1), which is expressed on T-cells, B-cells, and natural killer (NK) cells, is a transmembrane protein that acts as an immune checkpoint receptor. ${ }^{1,2}$ Two PD-1 ligands that have been identified are pro-

*Hye Won Lee and Kyung Joo Cho have equally contributed to this work. 
grammed death-ligand 1 (PD-L1) and programmed deathligand 2 (PD-L2). ${ }^{3} \mathrm{PD}-\mathrm{L} 1$ is expressed by tumor cells and is up-regulated in several malignancies. ${ }^{2,4-6}$ The interaction of PD-1 with PD-L1 results in the suppression of antitumor adaptive responses and immune escape of tumor cells. ${ }^{7-9}$ This ultimately leads to poor prognosis in patients with malignancies. The blockade of immune checkpoints using monoclonal antibodies targeting the PD-1/PD-L1 pathway has shown promising results, especially in advanced solid tumors. ${ }^{10}$ Elevated levels of soluble PD-L1 have been associated with poor prognosis in patients with renal cell carcinoma and in those with multiple myeloma. ${ }^{11,12}$

Hepatocellular carcinoma (HCC) is the most common primary liver cancer, and its incidence is increasing. ${ }^{13}$ In patients with advanced HCC, the few options for treatment include sorafenib and regorafenib. The results of clinical trials targeting the PD-1 axis in HCC have been recently reported. ${ }^{14}$ The objective response rate was $20 \%$ and $15 \%$ in patients treated with nivolumab in phase $1 / 2$ dose-expansion and dose-escalation phases. ${ }^{14}$ In contrast, anti-PD-1 with nivolumab produced a disease control rate of $81.8 \%$, with an objective response rate of $63.6 \%$ in patients with advanced HCC. ${ }^{15}$ The prognostic role of PD-1/PD-L1 in HCC is not well understood. One study showed that high expression of PD-L1 was associated with poor outcome in patients with HCC. ${ }^{1,16}$ In contrast, PD-L1 expression was not correlated directly with the survival of patients in the surgery alone group. ${ }^{17}$ Thus, in patients with HCC, questions remain of whether PD-L1 levels in tissue (tPD-L1) are correlated with serum PD-L1 (sPD-L1)/serum PD-1 (sPD-1) levels, and whether a correlation exists with prognosis.

In this study, we investigated the levels of tPD-L1, sPD-L1, and sPD-1 in samples from patients with HCC. In addition, we aimed to evaluate the clinical significance of PD-1 and PD-L1 levels in patients with HCC.

\section{METHODS}

\section{Patients}

We enrolled patients with HCC who underwent surgical resection between 2012 and 2017 at Severance Hospital, Seoul, Korea. The diagnosis of HCC was made according to current guidelines, ${ }^{18}$ and matched tissue and serum samples were obtained from patients. The exclusion criteria were age $\leq 18$ years, history of malignancy other than HCC, and history of liver transplantation. Ten serum samples from a healthy control cohort were retrieved from Yonsei Liver Blood Bank. In addition, serial serum samples were collected from 9 patients with HCC undergoing nivolumab treatment. Patients received $240 \mathrm{mg}$ nivolumab intravenously every 2 weeks. This study was approved by the Independent Institutional Review Board of Severance Hospital (IRB number: 4-20161107 ) and conformed to the ethical guidelines of the 1975 Declaration of Helsinki.

\section{Collection of tissue and blood samples}

The tissue and blood specimens were obtained from a biobank at Severance Hospital, Seoul, Korea. Primary HCC tissues $(n=78)$ and matching non-neoplastic tissue were collected immediately following surgery and stored at $-80^{\circ} \mathrm{C}$ until processing and use. On the day of surgery, blood samples were obtained from each patient. The serum was obtained by centrifugation $(3,000 \times \mathrm{g}$ for 10 minutes $)$ and stored at $-80^{\circ} \mathrm{C}$ until analysis.

\section{Immunohistochemistry and Enzyme-Linked Immunosorbent Assay (ELISA) assay}

The quantification of PD-L1 expression and tumor-infiltrating lymphocytes (TIL), including cluster of differentiation (CD)3 and CD8, were analyzed by immunohistochemical detection. The tissue samples were immersed overnight in freshly prepared 10\% neutral-buffered formalin. Fixed tissue samples were embedded in paraffin and sectioned serially into $6 \mu \mathrm{m}$ slices. The slices were stained with hematoxylin and eosin. The paraffin sections were deparaffinized in $x y-$ lene and rehydrated in gradually decreasing ethanol concentrations. The antigen epitopes were then unmasked using sodium citrate buffer $(6.0 \mathrm{pH})$. Subsequently, the sections were incubated overnight at $4{ }^{\circ} \mathrm{C}$ with the primary antibody PD-L1 
(13684S; Cell Signaling Technology, Inc., Danvers, MA, USA). After primary antibody incubation, the sections were incubated with the appropriate biotinylated secondary antibody (PK-6101; Vector Laboratories Inc, Burlingame, CA, USA) followed by treatment with freshly prepared 3,3'-diaminobenzidine substrate (SK-4100; Vector Laboratories Inc). The sections were lightly counter-stained with hematoxylin and mounted.

The expression levels of PD-L1 in HCC tissues were scored. Scores were determined based on the percentage of positively stained immunoreactive cells and the staining intensity in tumor cells (score 1, no staining; score 2, <10\% and weak staining; score 3, 10-50\% and moderate staining; and score $4,>50 \%$ and strong staining; Fig. 1). ${ }^{16}$

The serum samples were assayed by using commercially available ELISA kits for PD-1 and PD-L1 (Invitrogen, Corp., Carlsbad, CA, USA), following the manufacturer's protocol. Briefly, 96-well plates were incubated with standards at different concentrations, and serum samples were incubated for 2 hours at $37^{\circ} \mathrm{C}$. After covering of the biotinylated antibodies and several aspiration/wash cycles, the plates were incubated with horseradish peroxidase-conjugated streptavidin at $37^{\circ} \mathrm{C}$ for 1 hour and protected from light. Enzymatic reactions, as indicated by blue coloration on the addition of the substrate solution were developed and the absorbance was measured at $450 \mathrm{~nm}$ (A450) in a VersaMax ELISA microplate reader (Molecular Devices, San Jose, CA, USA). The protein levels were calculated according to standard curves. To properly evaluate the results, parallel investigations were made using healthy untreated controls. The minimum detectable level of
sPD-L1 was $31.621 \mathrm{ng} / \mathrm{mL}$. The level of soluble sPD-L1 was determined as described above, and the minimum detectable level of sPD-1 was $10.915 \mathrm{ng} / \mathrm{mL}$.

\section{Statistical analyses}

Continuous data are presented as median values (Q1-Q3), and categorical data are presented as counts and percentages. For continuous data, Student's $t$-test was used to test for differences between groups. When the assumption of normality was violated, a nonparametric test (Mann-Whitney test or Wilcoxon signed-rank test) was performed. Differences between categorical variables were examined for statistical significance chi-squared test (or Fisher's exact test, if appropriate). A two-sided $P$-value $<0.05$ was considered to indicate statistical significance. Pearson's chi-squared test and Fisher' s exact test were used for categorical data. Spearman's correlation coefficient was used to assess the association between the levels of $\mathrm{tPD}-\mathrm{L} 1$ and sPD-L1/sPD-1. The trend between sPD-L1/sPD-1 and tPD-L1 levels was tested using the Jonckheere-Terpstra test. Cox proportional-hazard models were performed to estimate the risk factors for overall survival. Cut-off values for sPD-L1/sPD-1 levels were calculated using the Contal and O'Quigley method. All analyses were performed using SAS software (version 9.4; SAS Institute Inc., Cary, NC, USA).
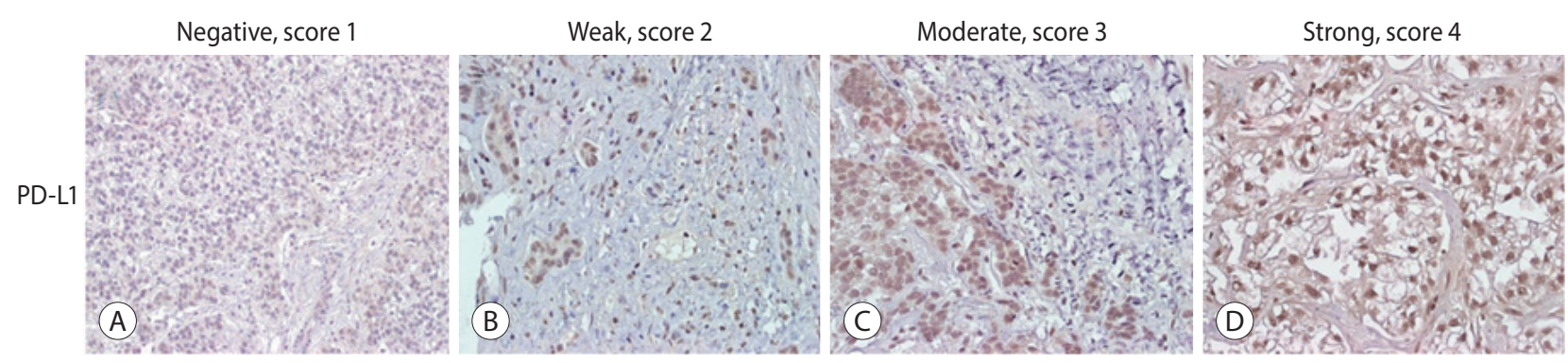

Figure 1. Immunohistochemical staining of hepatocellular carcinoma (HCC) tissues using anti-PD-L1. Representative staining patterns of HCC with negative, score $1(A)$, weak intensity, score 2 (B), moderate intensity, score $3(C)$, and strong intensity, score 4 (D) staining of PD-L1 are shown ( $\times 400)$. PD-L1, programmed cell death ligand 1 . 


\section{RESULTS}

\section{Baseline characteristics}

A total of 72 patients with HCC were included in this study. The baseline characteristics of the study population are summarized in Table 1. The median age was 58.5 years and male sex predominated (80.6\%). The etiologies of liver disease were hepatitis $B(n=53,73.6 \%)$, nonalcoholic steatohepatitis ( $\mathrm{n}=13,18.1 \%)$, hepatitis $\mathrm{C}(\mathrm{n}=4,5.6 \%)$, and others $(\mathrm{n}=2,2.8 \%)$. The median alanine aminotransferase and platelet levels were $27.0 \mathrm{IU} / \mathrm{mL}$ and $147 \times 10^{3} / \mu \mathrm{L}$, respectively. The median prothrombin time (internationalized ratio) was 1.0 and the median value of alpha-fetoprotein (AFP) was $12.6 \mathrm{ng} / \mathrm{mL}$. The tPD-L1 score was 1 in two (2.8\%) patients, 2 in $16(22.2 \%)$ patients, 3 in $27(37.5 \%)$ patients, and 4 in

Table 1. Baseline characteristics of the study population

\begin{tabular}{lc}
\hline Variable & Value \\
\hline Age (years) & $58.5(51.3-64.0)$ \\
Male & $58(80.6)$ \\
Etiology of liver disease & \\
Hepatitis B & $53(73.6)$ \\
Hepatitis C & $4(5.6)$ \\
NASH & $13(18.1)$ \\
Others & $2(2.8)$ \\
Laboratory findings & \\
Alanine aminotransferase (IU/dL) & $27.0(21.0-47.3)$ \\
Platelet $\left(10^{3} / \mu L\right)$ & $147(113-184)$ \\
PT (INR) & $1.0(1.0-1.1)$ \\
AFP $(\mathrm{ng} / \mathrm{mL})$ & $12.6(3.8-386.0)$ \\
tPD-L1 & \\
Score 1 & $2(2.8)$ \\
Score 2 & $16(22.2)$ \\
Score 3 & $27(37.5)$ \\
Score 4 & $27(37.5)$ \\
sPD-L1 (pg/mL) & $25.72(18.51-67.02)$ \\
sPD-1 (pg/mL) & $341.44(152.59-584.51)$ \\
\hline
\end{tabular}

Values are presented as median (Q1-Q3) or number (\%) NASH, nonalcoholic steatohepatitis; PT, prothrombin time; INR, international normalized ratio; AFP, alpha-fetoprotein; tPD-L1, PD-L1 levels in tissue; sPD-L1, PD-L1 in serum; sPD-1, PD-1 in serum; PD-1, programmed death-ligand 1.
$27(37.5 \%)$ patients.

\section{Levels of SPD-L1 and SPD-1}

The median sPD-L1 level was $25.72 \mathrm{pg} / \mathrm{mL}$ (Q1-Q3, $18.51-67.02 \mathrm{pg} / \mathrm{mL}$ ). The optimal cut-off values for the prediction of survival and HCC recurrence were $19.18 \mathrm{pg} / \mathrm{mL}$ and $34.03 \mathrm{pg} / \mathrm{mL}$, respectively. To define the sPD-L1 reference levels, sPD-L1 concentrations were determined from the serum of healthy blood donors. The median sPD-L1 level from 10 healthy donors (three male and seven female) with a median age of 28.5 years (Q1-Q3, 25.5-30.8 years) was 1.88 $\mathrm{pg} / \mathrm{mL}$ (Q1-Q3, 0.53-4.09 pg/mL).

The median sPD-1 level was $341.44 \mathrm{pg} / \mathrm{mL}$ (interquartile range, $152.59-584.51 \mathrm{pg} / \mathrm{mL}$ ). The optimal cut-off values for the prediction of survival and HCC recurrence were 279.10 $\mathrm{pg} / \mathrm{mL}$ and $719.64 \mathrm{pg} / \mathrm{mL}$, respectively. The median value from healthy donors was $28.5 \mathrm{pg} / \mathrm{mL}$ (Q1-Q3, 25.5-30.8 $\mathrm{pg} / \mathrm{mL}$ ). No difference was found between sexes. A positive correlation was detected between TPD-L1 and SPD-1 in patients with HCC $\left(R^{2}=0.430, P<0.001\right)$. The median sPD-1 level increased linearly with the tPD-L1 score $(P=0.002$; Fig. 2$)$.

\section{Liver-related mortality and recurrence of HCC}

During the follow-up period (median, 16.1 months), liverrelated mortality occurred in eight (10.3\%) patients. No sig-

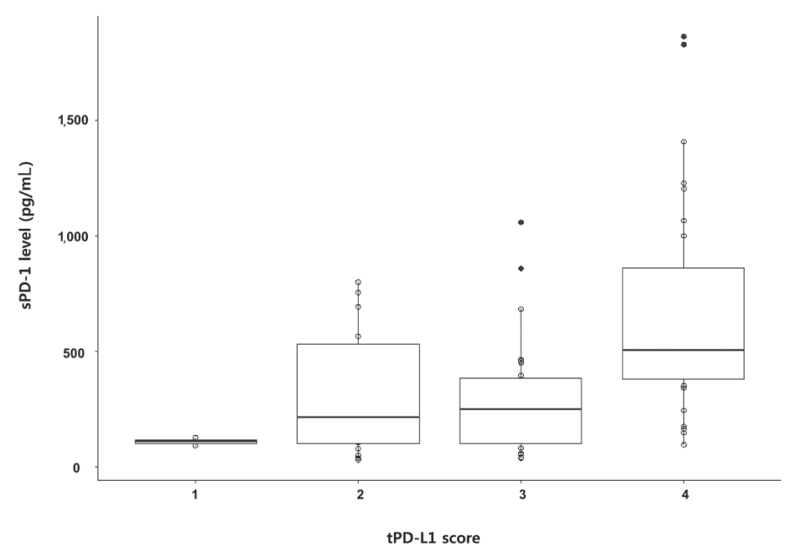

Figure 2. Linear trend between $\mathrm{PPD}-\mathrm{L} 1$ and SPD-1 levels. tPD-L1, PDL1 levels in tissue; SPD-1, PD-1 in serum; PD-1, programmed deathligand 1. 
nificant difference was observed in the baseline characteristics, except for the tPD-L1 score in patients with and without liver-related mortality (Table 2). The tPD-L1 scores were significantly higher in patients who died than in those who survived (tPD-L1 score $\geq 3,100 \%$ vs. $71.9 \%, P=0.035$ ).

HCC recurred in eight (10.3\%) patients during the followup. No difference was observed between patients with and without HCC recurrence (Table 3). In addition, no significant association was observed between the sPD-L1/sPD-1 level and recurrence of HCC (data not shown).

\section{Immunohistochemical analysis of TIL}

An immunohistochemical assessment of TILs was performed in an attempt to evaluate the contribution of relevant cells in cancer immune landscape, including cytotoxic, helper, and regulatory T-cells and NK cells. Considering the close relationship between $\mathrm{CD} 3+$ cell intensity and $\mathrm{PDD}-\mathrm{L} 1$ expression, we found lower PD-L1 expression together with negative $\mathrm{CD} 3+$ infiltration in tumor and higher PD-L1 expression accompanied with positive CD3+ infiltration. A significant correlation was detected between PD-L1 expression and

Table 2. Comparison of patients without and with liver-related mortality

\begin{tabular}{|c|c|c|c|}
\hline Variable & $\begin{array}{l}\text { Patients without liver-related } \\
\text { mortality }(n=64)\end{array}$ & $\begin{array}{l}\text { Patients with liver- } \\
\text { related mortality }(n=8)\end{array}$ & $P$-value \\
\hline Age (year) & $57.7 \pm 10.6$ & $59.5 \pm 12.8$ & 0.654 \\
\hline Male & $51(79.7)$ & $7(87.5)$ & 0.180 \\
\hline Etiology of liver disease & & & 0.173 \\
\hline Hepatitis B & $48(75.0)$ & $5(62.5)$ & \\
\hline Hepatitis C & $3(4.7)$ & $1(12.5)$ & \\
\hline NASH & $12(18.8)$ & $1(12.5)$ & \\
\hline Others & $1(1.6)$ & $1(12.5)$ & \\
\hline \multicolumn{4}{|l|}{ Laboratory findings } \\
\hline Alanine aminotransferase (IU/dL) & $27(21-44)$ & $36(29-64)$ & 0.180 \\
\hline Platelet $\left(10^{3} / \mu \mathrm{L}\right)$ & 147 (117-180) & $140(98-216)$ & 0.886 \\
\hline PT (INR) & $1.0 \pm 0.1$ & $1.0 \pm 0.1$ & 0.245 \\
\hline $\operatorname{AFP}(n g / m L)$ & $13.8(3.7-365.9)$ & $9.9(6.0-3623.9)$ & 0.792 \\
\hline tPD-L1 & & & 0.035 \\
\hline Score 1 & $2(3.1)$ & $0(0)$ & \\
\hline Score 2 & $16(25)$ & $0(0)$ & \\
\hline Score 3 & $20(31.3)$ & $7(87.5)$ & \\
\hline Score 4 & $26(40.6)$ & $1(12.5)$ & \\
\hline sPD-L1 (pg/mL) & $25.79(18.45-75.32)$ & $21.13(19.30-31.16)$ & 0.331 \\
\hline sPD-1 (pg/mL) & $347.08(158.24-586.67)$ & $260.3(146.91-494.37)$ & 0.674 \\
\hline \multicolumn{4}{|l|}{ Pathological findings } \\
\hline Fibrosis stage 4 & $27(42.2)$ & $3(37.5)$ & 0.834 \\
\hline PVI & $8(12.5)$ & $2(25.0)$ & 0.307 \\
\hline $\mathrm{HVI}$ & $1(1.6)$ & $1(12.5)$ & 0.211 \\
\hline MVI & $31(48.4)$ & $3(37.5)$ & 0.714 \\
\hline
\end{tabular}

Values are expressed as mean \pm standard deviation, median (Q1-Q3), or number (\%).

NASH, nonalcoholic steatohepatitis; PT, prothrombin time; INR, international normalized ratio; AFP, alpha-fetoprotein; tPD-L1, PD-L1 levels in tissue; SPD-L1, PD-L1 in serum; SPD-1, PD-1 in serum; PD-1, programmed death-ligand 1; PVI, portal vein invasion; HVI hepatic vein invasion; MVI, microvascular invasion. 
$\mathrm{CD} 8+$ lymphocytes and was the same as the correlation between PD-L1 expression and CD3+ lymphocytes (Supplementary Fig. 1).

\section{Independent factors for survival in patients with $\mathrm{HCC}$}

A multivariate analysis showed that higher SPD-L1 levels (cut-off value, $\geq 19.2 \mathrm{pg} / \mathrm{mL}$ ) tended to be associated with liver-related mortality (hazard ratio [HR], 6.866; 95\% confidence interval, 0.804-58.659), but this association did not reach statistical significance $(P=0.078$; Table 4$)$.

\section{Changes of SPD-1 level in patients with ad- vanced $\mathrm{HCC}$ treated with nivolumab}

Serially collected serum samples from a total of nine patients with advanced HCC who received nivolumab treatment were included for analysis (Supplementary Table 1). The median age was 60.0 years, and male sex predominated (88.9\%). All patients had extrahepatic metastasis. The mean number of nivolumab administrations was 9.4 (range, 6-13).

Table 3. Comparison of patients without and with hepatocellular carcinoma recurrence

\begin{tabular}{|c|c|c|c|}
\hline Variable & $\begin{array}{c}\text { Patients without } \\
\text { HCC recurrence }(n=64)\end{array}$ & $\begin{array}{c}\text { Patients with } \\
\text { HCC recurrence }(n=8)\end{array}$ & $P$-value \\
\hline Age (year) & $58.1 \pm 11.1$ & $56.3 \pm 8.1$ & 0.654 \\
\hline Male & $51(79.7)$ & $7(87.5)$ & $>0.999$ \\
\hline Etiology of liver disease & & & 0.683 \\
\hline Hepatitis B & 47 (73.4) & $6(75)$ & \\
\hline Hepatitis C & $3(4.7)$ & $1(12.5)$ & \\
\hline $\mathrm{NASH}$ & $12(18.8)$ & $1(12.5)$ & \\
\hline Others & $2(3.1)$ & $0(0)$ & \\
\hline \multicolumn{4}{|l|}{ Laboratory findings } \\
\hline Alanine aminotransferase (IU/mL) & $28(22-48)$ & $24(19-33)$ & 0.267 \\
\hline Platelet $\left(10^{3} / \mu \mathrm{L}\right)$ & $143(115-180)$ & $162(114-234)$ & 0.513 \\
\hline PT (INR) & $1.0 \pm 0.1$ & $1.0 \pm 0.1$ & 0.798 \\
\hline $\operatorname{AFP}(\mathrm{ng} / \mathrm{mL})$ & $13.5(3.7-392.7)$ & $12.0(7.9-1443.3)$ & 0.592 \\
\hline tPD-L1 & & & 0.304 \\
\hline Score 1 & $2(3.1)$ & $0(0)$ & \\
\hline Score 2 & $16(25.0)$ & $0(0)$ & \\
\hline Score 3 & $22(34.4)$ & $5(62.5)$ & \\
\hline Score 4 & $24(37.5)$ & $3(37.5)$ & \\
\hline sPD-L1 (pg/mL) & $25.72(18.45-70.10)$ & $24.79(19.89-30.20)$ & 0.750 \\
\hline sPD-1 (pg/mL) & 341.44 (141.37-546.03) & $356.96(214.17-860.85)$ & 0.457 \\
\hline \multicolumn{4}{|l|}{ Pathological findings } \\
\hline Fibrosis stage 4 & $26(40.6)$ & $4(50.0)$ & $>0.999$ \\
\hline PVI & $8(12.5)$ & $2(25.0)$ & 0.307 \\
\hline $\mathrm{HVI}$ & $2(3.1)$ & $0(0)$ & $>0.999$ \\
\hline MVI & $31(48.4)$ & $3(37.5)$ & 0.714 \\
\hline
\end{tabular}

Values are presented as mean \pm standard deviation, median (Q1-Q3), or number (\%).

HCC, hepatocellular carcinoma; NASH, nonalcoholic steatohepatitis; PT, prothrombin time; INR, international normalized ratio; AFP, alphafetoprotein; tPD-L1, PD-L1 levels in tissue; SPD-L1, PD-L1 in serum; SPD-1, PD-1 in serum; PD-1, programmed death-ligand 1; PVI, portal vein invasion; HVI, hepatic vein invasion; MVI, microvascular invasion. 
Table 4. Independent factors for survival in patients with hepatocellular carcinoma

\begin{tabular}{lcc}
\hline Variable & Hazard ratio $\mathbf{9 5 \%} \mathrm{Cl})$ & $\boldsymbol{P}$-value \\
\hline Age (years) & $1.016(0.955-1.080)$ & 0.619 \\
Female & $0.670(0.081-5.516)$ & 0.710 \\
SPD-L1 $\geq 19.181 \mathrm{pg} / \mathrm{mL}$ & $6.866(0.804-58.659)$ & 0.078 \\
SPD-1 $\geq 279.104 \mathrm{pg} / \mathrm{mL}$ & $0.437(0.103-1.859)$ & 0.262 \\
Platelet $\left(10^{3} / \mu \mathrm{L}\right)$ & $0.999(0.098-1.012)$ & 0.913 \\
AFP $(\mathrm{ng} / \mathrm{mL})$ & $2.328(0.555-9.763)$ & 0.624 \\
Fibrosis stage 4 & $0.563(0.058-5.457)$ & 0.620 \\
PVI & $5.265(0.957-28.975)$ & 0.056 \\
HVI & $10.788(1.107-105.154)$ & 0.041
\end{tabular}

Cl, confidence interval; sPD-L1, PD-L1 in serum; SPD-1, PD-1 in serum; PD-1, programmed death-ligand 1; AFP, alpha-fetoprotein; PVI, portal vein invasion; $\mathrm{HVI}$, hepatic vein invasion.

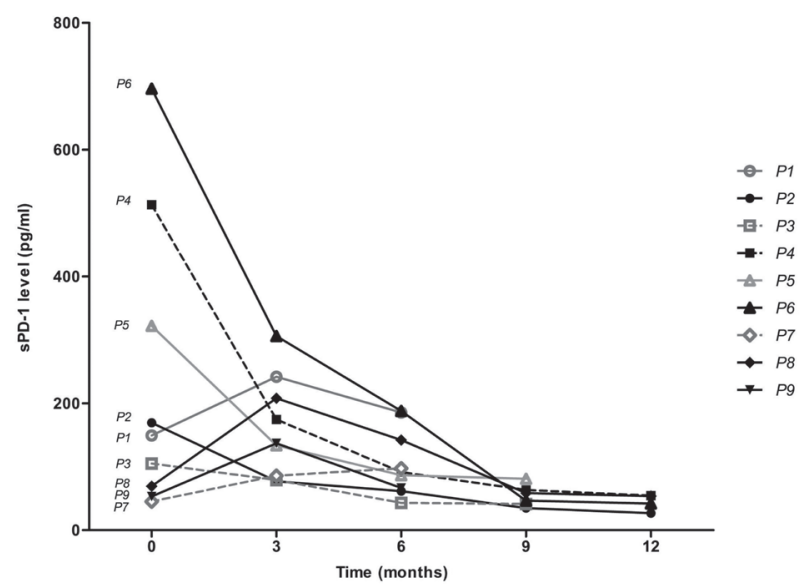

Figure 3. P1-P9 refers to each patient case. Change of sPD-1 levels according to nivolumab treatment. sPD-1, PD-1 in serum; PD-1, programmed death-ligand 1.

The changes in PD-1 are shown in Fig. 3. Of the patients who responded to nivolumab treatment, the best responses during the treatment were categorized as partial response (PR; $\mathrm{n}=2,22.2 \%$ ), stable disease (SD; $\mathrm{n}=3,33.3 \%$ ), and progressive disease (PD; $\mathrm{n}=4,44.5 \%)$. Except for one of the five favorable therapeutic responses, a reduction of $\mathrm{SPD}-1$ by $\geq$ $50 \%$ compared with baseline was observed immediately after nivolumab administration. One of the favorable therapeutic responses showed a serial decrease after 9 doses but eventually showed PD. The final responses were PR and SD in one patient each after the administration of 12 doses, and these patients ultimately developed PD. However, no change in the
sPD-L1 level that matched the therapeutic response was observed (Supplementary Fig. 2).

\section{DISCUSSION}

PD-1 inhibits T-cell proliferation, survival, and function. Anti-PD-1 drugs have shown dramatic treatment results for patients with melanoma and lung cancer. A recent phase I/II trial showed that nivolumab had a manageable safety profile and promising results were obtained in patients with advanced HCC. ${ }^{14}$ The response rate was $15-20 \%$ in patients treated with nivolumab. However, few studies have examined the prognostic roles of PD-L1/PD-1 in HCC. Thus, we investigated PD-L1/PD-1 levels and their clinical significance in patients with HCC.

In another study, the median sPD-L1 levels were $500 \mathrm{pg} / \mathrm{mL}$ (range, 30-6,040 pg/mL) in patients with HCC and $780 \mathrm{pg} / \mathrm{mL}$ (range, $168-1,521 \mathrm{pg} / \mathrm{mL}$ ) in healthy controls. ${ }^{1}$ In our study, the result was $25 \mathrm{pg} / \mathrm{mL}$, which was relatively low. This difference may have been due to the inclusion of patients with HCC at various stages (Barcelona Clinic Liver Cancer stages A-D) in the other study. In contrast, only patients with HCC at early stages who underwent surgery were included in our study. The median sPD-1 levels were 341 pg/mL (Q1-Q3, $153-585 \mathrm{pg} / \mathrm{mL}$ ) in patients with HCC and $29 \mathrm{pg} / \mathrm{mL}$ (Q1Q3, 26-31 pg/mL) in healthy controls.

No strong biomarker predicting postoperative outcome in 
HCC has been identified. In some studies, a preoperative serum AFP level (>400 ng/mL) was an independent prognostic factor for postoperative HCC survival. ${ }^{19,20}$ However, in our study, higher AFP level $(>400 \mathrm{ng} / \mathrm{mL})$ was not associated with prognosis in patients with HCC. In addition, we found no correlation between the AFP and PD-L1/PD-1 levels (data not shown). PD-L1 expression has been associated with poor prognosis in malignancies, including melanoma, non-small cell lung cancer, esophageal cancer, gastric cancer, pancreatic cancer, renal cell cancer, and ovarian cancer. ${ }^{21-23}$ Use of the PD-L1 level as a prognostic factor in patients with HCC has also been suggested. ${ }^{1}$ High sPD-L1 levels were associated with mortality, independent of the stage of cirrhosis, levels of AFP and a macrophage activation marker. A characterization of tumor-infiltrating T-cell and macrophage subsets, including their expression of PD-1 and PD-L1, has been suggested for future biomarker assessment in patients with HCC. ${ }^{14}$ However, we were unable to identify a prognostic role of PDL1/PD-1 in patients with HCC.

sPD-1 levels in patients treated with nivolumab showed significant changes according to the treatment response. Four patients with best responses showed dramatic (>50\%) reductions in their SPD-L1 levels immediately after nivolum$\mathrm{ab}$ administration compared with baseline. However, these patients developed PD as treatment continued. Tumor cells can directly escape T-cell recognition by down-regulating major histocompatibility class class I expression and up-regulating the expression of PD-L1, CTLA-4, and other ligands of inhibitory T-cell receptors that mediate T-cell exhaustion. ${ }^{24} \mathrm{NK}$ cells may also affect the immune response in advanced HCC independently of T-cells; further studies are needed to explore this possibility.

Our study had several strengths. First, we measured the levels of PD-L1 in tissues and PD-L1/PD-1 in sera and investigated their association. Second, PD-1/PD-L1 level was checked in a small number of patients, but these reference values can be used for future research. Third, sera from patients who received anti-PD-1 treatment were collected serially, and the relationship of levels therein to prognosis was investigated. Lastly, we found that the PD-L1/PD-1 levels changed based on the circumstances and enhanced immune responses against tumors. However, we confirmed that the PD-L1/PD-1 level did not predict the clinical outcome in patients with early-stage HCC.

We are also aware of several issues in our study that remain unresolved. The limitations include the small sample size and the inclusion of only patients who received curative treatment. However, the collection of blood and tissue samples at the time of surgery allowed us to examine the samples at the proper time. This approach was advantageous because it allowed us to acquire and analyze patient information, even though the study was retrospective. Further studies involving large numbers of patients and examining the roles of $\mathrm{T}$-cells and NK cells in patients receiving immunotherapy are warranted. In conclusion, PD-1/PD-L1 levels changed according to immunotherapy, but no significant association with clinical outcomes was found in patients with early-stage HCC.

\section{ACKNOWLEDGMENT}

The authors are grateful to Hye Mi Bae (medical record reviewer, Yonsei Liver Center, Yonsei University College of Medicine, Seoul, Korea) for the help with this paper.

\section{GRANT SUPPORT}

This work was supported by an academic research fund of the Korean Liver Cancer Association for 2014.

\section{SUPPLEMENTARY MATERIAL}

Supplementary datas can be found with this article online http://www.e-jlc.org/html/https://doi.org/10.17998/ jlc.19.2.108.

\section{Conflicts of Interest}

The authors have no conflicts to disclose.

\section{REFERENCES}

1. Finkelmeier F, Canli Ö, Tal A, Pleli T, Trojan J, Schmidt M, et al. 
High levels of the soluble programmed death-ligand (SPD-L1) identify hepatocellular carcinoma patients with a poor prognosis. Eur J Cancer 2016:59:152-159.

2. Uhercik M, Sanders AJ, Owen S, Davies EL, Sharma AK, Jiang WG, et al. Clinical significance of PD1 and PDL1 in human breast cancer. Anticancer Res 2017;37:4249-4254.

3. Woo SR, Turnis ME, Goldberg MV, Bankoti J, Selby M, Nirschl CJ, et al. Immune inhibitory molecules LAG-3 and PD-1 synergistically regulate T-cell function to promote tumoral immune escape. Cancer Res 2012;72:917-927.

4. Zheng P, Zhou Z. Human cancer immunotherapy with PD-1/PD-L1 blockade. Biomark Cancer 2015;7(Suppl 2):15-18.

5. Zhao T, Li C, Wu Y, Li B, Zhang B. Prognostic value of PD-L1 expression in tumor infiltrating immune cells in cancers: a metaanalysis. PLoS One 2017;12:e0176822.

6. Wang C, Zhu H, Zhou Y, Mao F, Lin Y, Pan B, et al. Prognostic value of PD-L1 in breast cancer: a meta-analysis. Breast J 2017;23:436-443.

7. Waidmann 0, Trojan J. Novel drugs in clinical development for hepatocellular carcinoma. Expert Opin Investig Drugs 2015;24:10751082.

8. Disis ML. Immune regulation of cancer. J Clin Oncol 2010;28:45314538.

9. Miller JF, Sadelain M. The journey from discoveries in fundamental immunology to cancer immunotherapy. Cancer Cell 2015;27:439449.

10. Carretero-Gonzalez A, Lora D, Ghanem I, Zugazagoitia J, Castellano $D$, Sepúlveda JM, et al. Analysis of response rate with ANTI PD1/PD-L1 monoclonal antibodies in advanced solid tumors: a meta-analysis of randomized clinical trials. Oncotarget 2018;9:87068715.

11. Frigola X, Inman BA, Lohse CM, Krco CJ, Cheville JC, Thompson $\mathrm{RH}$, et al. Identification of a soluble form of B7-H1 that retains immunosuppressive activity and is associated with aggressive renal cell carcinoma. Clin Cancer Res 2011;17:1915-1923.

12. Zhou J, Mahoney KM, Giobbie-Hurder A, Zhao F, Lee S, Liao X, et al. Soluble PD-L1 as a biomarker in malignant melanoma treated with checkpoint blockade. Cancer Immunol Res 2017;5:480-492.

13. Sayiner M, Golabi P, Younossi ZM. Disease burden of hepatocellu- lar carcinoma: a global perspective. Dig Dis Sci 2019;64:910-917.

14. El-Khoueiry AB, Sangro B, Yau T, Crocenzi TS, Kudo M, Hsu C, et al. Nivolumab in patients with advanced hepatocellular carcinoma (CheckMate 040): an open-label, non-comparative, phase 1/2 dose escalation and expansion trial. Lancet 2017;389:2492-2502.

15. Feng D, Hui X, Shi-Chun L, Yan-Hua B, Li C, Xiao-Hui L, et al. Initial experience of anti-PD1 therapy with nivolumab in advanced hepatocellular carcinoma. Oncotarget 2017;8:96649-96655.

16. Jung HI, Jeong D, Ji S, Ahn TS, Bae SH, Chin S, et al. Overexpression of PD-L1 and PD-L2 is associated with poor prognosis in patients with hepatocellular carcinoma. Cancer Res Treat 2017;49:246-254.

17. Chen $C L$, Pan QZ, Zhao JJ, Wang Y, Li YQ, Wang QJ, et al. PD-L1 expression as a predictive biomarker for cytokine-induced killer cell immunotherapy in patients with hepatocellular carcinoma. Oncoimmunology 2016;5:e1176653.

18. Omata M, Cheng AL, Kokudo N, Kudo M, Lee JM, Jia J, et al. Asiapacific clinical practice guidelines on the management of hepatocellular carcinoma: a 2017 update. Hepatol Int 2017;11:317-370.

19. Ma WJ, Wang HY, Teng LS. Correlation analysis of preoperative serum alpha-fetoprotein (AFP) level and prognosis of hepatocellular carcinoma (HCC) after hepatectomy. World J Surg Oncol 2013;11:212.

20. Ikai I, Arii S, Kojiro M, Ichida T, Makuuchi M, Matsuyama Y, et al. Reevaluation of prognostic factors for survival after liver resection in patients with hepatocellular carcinoma in a Japanese nationwide survey. Cancer 2004;101:796-802.

21. Bilgin B, Sendur MA, Bülent Akıncı M, Şener Dede D, Yalçın B. Targeting the PD-1 pathway: a new hope for gastrointestinal cancers. Curr Med Res Opin 2017;33:749-759.

22. Guilleminault L, Carmier D, Heuzé-Vourc'h N, Diot P, Pichon E. Immunotherapy in non-small cell lung cancer: inhibition of PD1/PDL1 pathway. Rev Pneumol Clin 2015;71:44-56.

23. Philips GK, Atkins MB. New agents and new targets for renal cell carcinoma. Am Soc Clin Oncol Educ Book 2014;e222-e227.

24. Han Q, Wang Y, Pang M, Zhang J. STAT3-blocked whole-cell hepatoma vaccine induces cellular and humoral immune response against HCC. J Exp Clin Cancer Res 2017;36:156. 
Hye Won Lee, et al. Association between sPD1 and prognosis

Supplementary Table 1. Characteristics of nine patients who received nivolumab treatment

\begin{tabular}{|c|c|c|c|c|c|c|c|}
\hline Patient No. & Age & Gender & Etiology & Cirrhosis & AFP & Best response & Final response \\
\hline 1 & 60 & M & NBNC & No & 3,699 & PD & PD \\
\hline 2 & 55 & M & HBV & No & 2.5 & $P R$ & PD \\
\hline 3 & 57 & M & HBV & No & 2.0 & PD & PD \\
\hline 4 & 52 & M & HBV & No & 8,993 & SD & PD \\
\hline 5 & 65 & M & HBV & Yes & 329.2 & SD & PD \\
\hline 6 & 72 & $\mathrm{~F}$ & HBV & No & $>120,000$ & SD & SD \\
\hline 7 & 65 & $M$ & HBV & Yes & $2,253.0$ & PD & PD \\
\hline 8 & 77 & M & HBV & No & 476.5 & $P R$ & SD \\
\hline 9 & 51 & M & HBV & Yes & $>120,000$ & PD & PD \\
\hline
\end{tabular}

AFP, alpha-fetoprotein; M, male; NBNC, non-B non-C; PD, progressive disease; HBV, hepatitis B; PR, partial response; SD, stable disease; F, female. 


\section{tPD-L1 Negative}

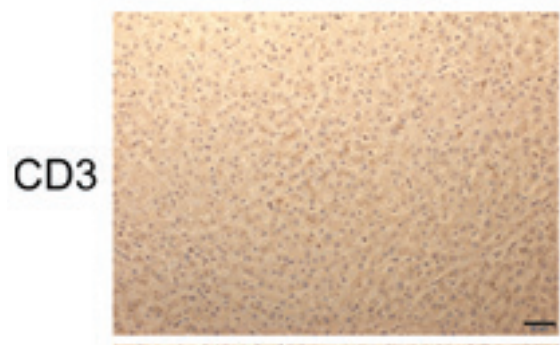

CD8

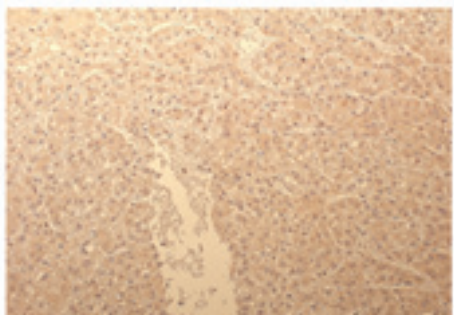

$\underline{\text { tPD-L1 Positive }}$
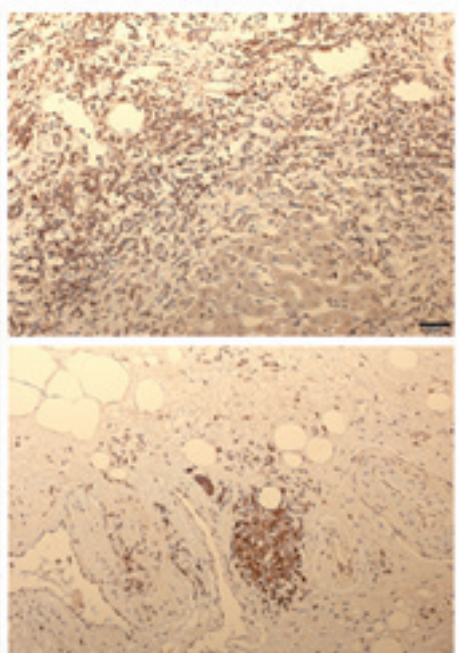

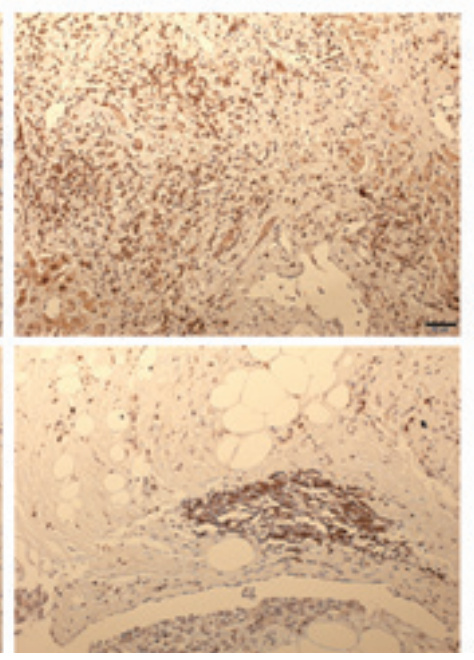

Supplementary Figure 1. Immunohistochemical staining of tumor-infiltrating immune cells; with total (CD3+) and cytotoxic (CD8+) T lymphocytes from tPD-L1 negative and positive HCC tissue samples (×100). CD, cluster of differentiation; tPD-L1, PD-L1 levels in tissue; PD-1, programmed death-ligand 1, HCC, hepatocellular carcinoma. 


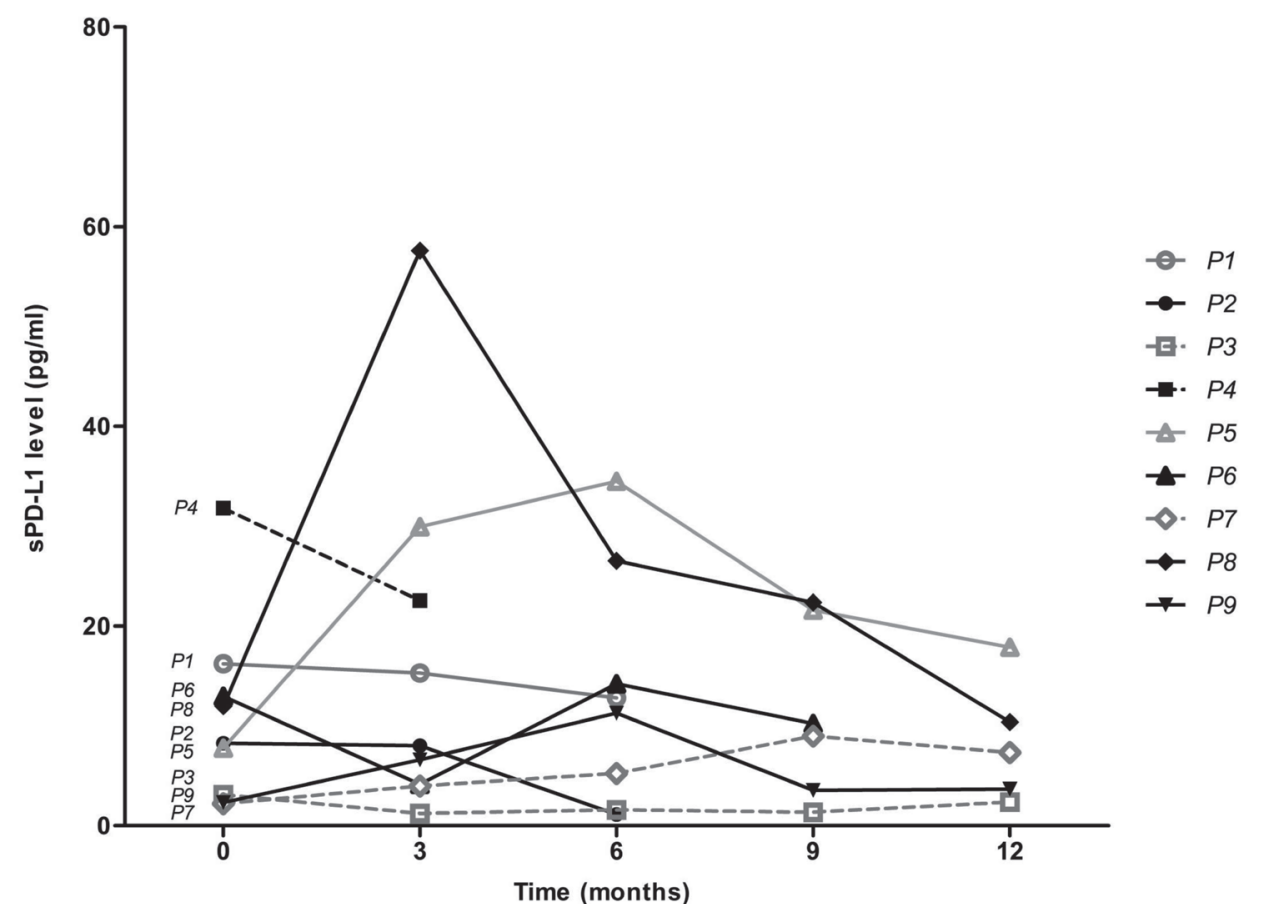

Supplementary Figure 2. Change of sPD-L1 levels according to nivolumab treatment. P1-P9 refers to each patient case. SPD-1, PD-1 in serum; PD-1, programmed death-ligand 1. 